\title{
Renormalized solution for Stefan type problems: existence and uniqueness
}

\author{
Noureddine Igbida, Karima Sbihi, and Petra Wittbold
}

\begin{abstract}
We consider a class of nonlinear degenerate problems of Stefan type:

$$
u_{t}-\Delta w-\nabla F(u, w)=g(\cdot, u), w \in \beta(u)
$$

where $\beta$ is a maximal monotone graph in $\mathbb{R}^{2}$, with homogenous Dirichlet conditions and initial conditions. Under rather general assumptions on $F$ and $g$, we prove existence and uniqueness of renormalized solutions.

Mathematics Subject Classification (2000). 34A1 · 35J60 - 35K65.

Keywords. Renormalized solution, Weak solution, Quasi-linear degenerate parabolic equation, Stefan problem, Nonlinear semigroup of contraction.
\end{abstract}

\section{Introduction}

Let $\Omega$ be a bounded open domain of $\mathbb{R}^{N}$ with smooth boundary, $T>0$. For given function $g$, and $u_{0} \in L^{1}(\Omega)$ we consider the evolution problem

$$
\left\{\begin{array}{lll}
u_{t}-\Delta w-\nabla F(u, w)=g(t, x, u), & w \in \beta(u) & \text { in } \quad Q:=(0, T) \times \Omega \\
w=0 & \text { on } \quad \Sigma:=(0, T) \times \partial \Omega \\
u(0, .)=u_{0}(.) & \text { in } \quad \Omega,
\end{array}\right.
$$

under the assumptions:

$\beta$ is a maximal monotone graph such that $0 \in \beta(0)$,

$$
\begin{gathered}
F\left(r_{1}, r_{2}\right)=F_{1}\left(r_{2}\right)+r_{1} F_{2}\left(r_{2}\right) \text { for any } r_{1}, r_{2} \in \mathbb{R} \\
\text { with } F_{i} \in C(\mathbb{R} ; \mathbb{R}) \text { and } F_{2}(0)=0,
\end{gathered}
$$

$$
\left(\begin{array}{l}
\text { i) } g(t, x, r) \text { is continuous in } r \text { and measurable in }(t, x) \\
\text { ii) } \frac{\partial g}{\partial r}(t, x, r) \leq C \text { in } \mathcal{D}^{\prime}(\mathbb{R}), C \in \mathbb{R}^{+} \\
\text {iii })|g(t, x, r)| \leq C_{1}(t, x)|r|+C_{2}(t, x)
\end{array}\right.
$$


with $C_{1}, C_{2} \in L^{1}(Q)$, There is an extensive literature on this type of problems, since it serves as a mathematical model for a large class of physical problems (see $[1,20]$ and the references therein). A large field of applications corresponds to the case of maximal montone graph $\beta$ (not continuous) such that $\beta^{-1}$ is continuous. for which there exists a large number of references. In particular, $E\left(u_{0}, g\right)$ models in this case free boundary problems involving a solid-liquid phase change of Stefan type for which there exists a large number of references. Among them, let us mention the earlier works $[1,17,19]$. A complete bibliography may be found in [30]. The structure condition $\left(H_{2}\right)$ includes in particular the Stefan problem with a temperature dependent convective term (see for instance $[31,32]$ ).

The problem of establishing uniqueness of solutions of $E\left(u_{0}, g\right)$ seems to be complicated in general. The equation in $E\left(u_{0}, g\right)$ has a hyperbolic character in the set where $w=0$, and we say that $E\left(u_{0}, g\right)$ is of parabolic-hyperbolic type; in general, uniqueness of a weak solution as well as uniqueness of renormalized solution do not hold. In [15], Carrillo proves that problems of type $E\left(u_{0}, g\right)$ are well posed using the concept of "entropy solutions", which are weak solutions that satisfy some additional conditions called entropy conditions. However, under the additional structure condition $\left(H_{2}\right)$, it is well known by now (see $[4,15,21,22])$ that Problem $E\left(u_{0}, g\right)$ is expected to admit at most one weak solution which, by definition, is a function $u \in L^{1}(Q)$ such that $w \in L^{2}\left(0, T ; H_{0}^{1}(\Omega)\right)$ and satisfies the equation in $\mathcal{D}^{\prime}(Q)$. As to the existence of a weak solution, this requires additional assumptions on the data $u_{0}$ and $g$, for instance $u_{0} \in L^{\infty}(\Omega)$ and $g \in L^{\infty}(Q)$. In this paper, we consider the case where all the right hand side data belongs to $L^{1}$. This means that all the sources should have finite energy, which is a physically reasonable requirement.

In order to solve $E\left(u_{0}, g\right)$ for general $L^{1}$-data one needs a more general notion of solution. The framework of renormalized solution, which was originally introduced in [18] for study the Boltzmann equation, has proved to be a powerful approach to study a large of class of problems, see, among others, $[3,6,11-14,25,27,29]$.

In the case where $\beta^{-1}$ is a nondecreasing continuous function, problem $E\left(u_{0}, g\right)$ is a particular case of the so-called elliptic-parabolic problem, and has been studied extensively in the literature (see $[1,3,10,23,26]$, and the references therein). For instance, if $F$ is continuous, it is proved among the results of [16], that, for any $u_{0} \in L^{1}(\Omega)$ and $g \in L^{1}(Q), E\left(u_{0}, g\right)$ has at most one renormalized solution. Existence of this type of solution has been shown in [3] (see also [13] where the case of a strictly increasing regular function $\beta$ is treated). The case where $\beta^{-1}$ is a nondecreasing multivalued function has been studied in $[28,29]$ where the authors established existence and uniqueness of renormalized solutions.

In this paper, we are interested in the case where $\beta$ is a maximal monotone graph in $\mathbb{R}^{2}$ with $0 \in \beta(0)$, where the convection term satisfies the structure condition $\left(H_{2}\right)$ and where the data $g$ satisfies Assumption $\left(H_{3}\right)$. We prove that, for any $u_{0} \in L^{1}(\Omega)$, the problem $E\left(u_{0}, g\right)$ is well posed in the renormalized sense. We first consider the case where $g$ is an integrable function $f$, then 
we deduce existence of renormalized solution for any $g$ satisfying Assumption $\left(H_{3}\right)$ by using the results of [9].

The proof of existence of renormalized solution consists of two steps: in a first step, for bounded data, we study the non-degenerate problem: $\left(E_{k}\right) u_{t}-$ $\Delta w-\nabla F(u, w)=f, w \in \beta_{k}(u)$ on $Q$ ( + homogenous Dirichlet boundary conditions and initial conditions), and then we pass to the limit with $k$. Here $\beta_{k}$ is an approximation of the graph $\beta$. Existence of weak solutions of this non-degenerate problem is ensured by the work of [15], thanks to the nonlinear semigroups theory (see $[7,8]$ ). In order to pass to the limit with $k$, we need $L^{\infty}$-estimates and strong convergence in $L^{1}$ of the sequence $\left(w_{k}\right)_{k}$ (see the proof of Proposition 4.2), which are not easy to obtain. To overcome this difficulty we add to Problem $\left(E_{k}\right)$ a monotone function $\psi_{m, n}(w)$. Recall that this type of arguments was already used in [2,3] for elliptic-parabolic problem, and in [5] for parabolic problem of absorption type. Due to the strongly monotone perturbation term, one can prove an $L^{1}$-estimate and, in particular, the strong compactness of the sequence of solutions $w$ and also its strong convergence in $L^{1}$ to a measurable function. This allows to pass to the limit with $k$ in Problem $\left(E_{k}\right)$ with a fixed perturbation $\psi_{m, n}$.

In the second step, using a bi-monotone approximation $u_{m, n}^{0}, f_{m, n}$ of the data $u_{0}, f$, in the same way of [3], we obtain a monotone sequence of weak solutions $u_{m, n}$ of Problem $E\left(u_{m, n}^{0}, f_{m, n}, \psi_{m, n}\right)$. For the convergence of $w_{m, n}, w_{m, n} \in \beta\left(u_{m, n}\right)$ (see the proof of Theorem 5.1) we use the monotonicity with respect to $m$ and $n$, and for the identification of the limit equation essential tool is the regularization method of Landes (see [24]).

The main difficulty when dealing with hyperbolic-parabolic problem is the uniqueness. In [15] the uniqueness of weak solutions was established under the additional assumption that $\beta^{-1}(0)=0$. In [21], the authors assumed that $F_{i}$ is Lipschitz continuous, and in [22], it is assumed that $F_{i}$ is continuous and satisfies $\|F(u, w)\| \leq C\|w\|^{2}$. Recently, [4] have proved uniqueness of weak solutions under only the structure condition $\left(H_{2}\right)$. In this paper, the uniqueness of renormalized solution is proved by using the result of [4], and the proof goes essentially as follows: we prove that, if $u$ is a renormalized solution of Problem $E\left(u_{0}, g\right)$, then $u$ is a weak solution of some degenerate parabolic problem (see the proof of Proposition 3.1), then, by using the comparison result of [4] of weak solutions, we deduce a comparison result of renormalized solutions, and also uniqueness.

Let us briefly summarize the contents of the paper: In Sect. 2 we fix the notations, give the concept of renormalized solution of Problem $E\left(u_{0}, g\right)$, and state the existence and uniqueness result for renormalized solution of Problem $E\left(u_{0}, g\right)$. In Sect. 3 we prove uniqueness of renormalized solutions by using the results of [4]. Section 4 is devoted to the study of a perturbed problem obtained by adding a monotone term. Existence of weak solution is proved for $L^{\infty}$-data. In Sect. 5 we give the proof of existence of a renormalized solution for Problem $E\left(u_{0}, g\right)$. It was shown that a weak solution of the perturbed problem $E\left(u_{0}, f, \psi_{m, n}\right)$ converges to a renormalized solution. Finally, in Sect. 6 we deduce the corresponding results for the associated stationary problem. 


\section{Preliminaries and main result}

In this section, after some notations, we introduce the concept of renormalized solution for Problem $E\left(u_{0}, g\right)$ and state the existence and uniqueness result for this type of solutions.

We denote by $|A|$ the Lebesgue measure of a set $A \subset \mathbb{R}^{N}$ and by $\chi_{A}$ the characteristic function of $A$. For $k \geq 0$, we denote by $T_{k}$ the truncation function at the level $k$, defined by

$$
T_{k}(u)=\left\{\begin{array}{lll}
k \operatorname{sign}_{0}(u) & \text { if } & |u|>k \\
u & \text { if } & |u| \leq k,
\end{array}\right.
$$

where $\operatorname{sign}_{0}(\cdot)$ denotes the single-valued function defined by $\operatorname{sign}_{0}(r)=-1$ if $r<0, \operatorname{sign}_{0}(r)=1$ if $r>0, \operatorname{sign}_{0}(r)=0$ if $r=0$. We denote by $\operatorname{sign}_{0}^{+}(\cdot)$ and $\operatorname{sign}_{0}^{-}(\cdot)$ the functions defined by $\operatorname{sign}_{0}^{+}(r)=1$ if $r>0,=0$ otherwise, and $\operatorname{sign}_{0}^{-}(r)=-1$ if $r<0,=0$ otherwise.

For $n \in \mathbb{N}$ we denote

$$
h_{n}(r)=\inf \left((n+1-|r|)^{+}, 1\right) \text { and } H_{n}(r)=\int_{0}^{r} h_{n}(s) d s .
$$

Throughout the paper, for the sake of simplicity, for $u$ a function of $(t, x)$ and for $k$ a real number, we denote, for example, $\{|u| \leq k\}$ the set $\{(t, x) \in$ $Q ;|u(t, x)| \leq k\}$. We also write $\int_{Q} u$ for $\int_{Q} u(t, x) d t d x$, etc... In the sequel $C$ denotes a constant that may change from line to line.

For a maximal monotone graph $\beta$ in $\mathbb{R} \times \mathbb{R}$; its main section $\beta_{0}$ is defined by

$$
\beta_{0}(r)= \begin{cases}\inf \beta(r) & \text { if } r>0 \\ 0 & \text { if } r=0 \\ \sup \beta(r) & \text { if } r<0\end{cases}
$$

with the usual convention $\inf \emptyset=+\infty$ and $\sup \emptyset=-\infty$.

An essential tool to prove existence of weak (renormalized) solutions is the following energy estimate similar to the one set of [1].

Let $j, \varphi: \mathbb{R} \rightarrow \mathbb{R}$ be a continuous, nondecreasing functions such that $j(0)=\varphi(0)=0$. For any continuous and monotone function $h$ we define the function

$$
B_{h}(s)= \begin{cases}\int_{0}^{s} h\left(\varphi \circ\left(j^{-1}\right)_{0}(r)\right) d r & \text { for } s \in \overline{(h \circ \varphi) \circ j^{-1}} \\ +\infty & \text { otherwise. }\end{cases}
$$

Lemma 2.1. [15, Lemma 4] Let $j, \varphi: \mathbb{R} \rightarrow \mathbb{R}$ be a continuous and nondecreasing function with $j(0)=\varphi(0)=0$. Let $v$ be a measurable function such that $j(v) \in L^{1}(Q), j(v)_{t} \in L^{2}\left(0, T ; H^{-1}(\Omega)\right)$ and $j(v)(0)=j\left(v_{0}\right)$, where $v_{0}: \Omega \rightarrow \mathbb{R}$ is measurable with $j\left(v_{0}\right) \in L^{1}(\Omega)$. Then

$$
B_{h}(j(v)) \in L^{\infty}\left(0, T ; L^{1}(\Omega)\right)
$$

and for a.e. $t \in[0, T]$

$$
\int_{\Omega} B_{h}\left(j(v(t)) \xi(t)-\int_{\Omega} B_{h}\left(j\left(v_{0}\right)\right) \xi(0)=\int_{0}^{t}\left\langle j(v)_{t}, h(\varphi(v)) \xi\right\rangle+\int_{0}^{t} \int_{\Omega} B_{h}(j(v)) \xi_{t}\right.
$$


for any $\xi \in C([0, T] \times \bar{\Omega})$ such that $h(\varphi(v)) \in L^{2}\left(0, T ; H_{0}^{1}(\Omega)\right)$, where $\langle\cdot, \cdot\rangle$ represents the duality product between $H^{-1}(\Omega)$ and $H_{0}^{1}(\Omega)$.

We now give the concept of renormalized solution for Problem $E\left(u_{0}, g\right)$.

Definition 2.1. Given $u_{0} \in L^{1}(\Omega)$ and $g: Q \times \mathbb{R} \rightarrow \mathbb{R}$ satisfying $\left(H_{3}\right)$, a renormalized solution of $E\left(u_{0}, g\right)$ is a function $u$ such that

(i) $u \in L^{1}(Q)$

(ii) there exists a measurable function $w$ such that $w \in \beta(u)$ a.e. on $Q$ and $T_{k} w \in L^{2}\left(0, T ; H_{0}^{1}(\Omega)\right)$ for any $k>0$

(iii) for all $\xi \in \mathcal{D}([0, T) \times \Omega)$ and $h \in \mathcal{C}_{c}^{1}(\mathbb{R})$

$$
-\int_{Q} \xi_{t} \int_{u_{0}}^{u} h\left(\beta_{0}(r)\right) d r+\int_{Q}(\nabla w+F(u, w)) \cdot \nabla(h(w) \xi)=\int_{Q} g(\cdot, u) h(w) \xi
$$

and moreover

$$
\int_{Q \cap\{n \leq|w| \leq n+1\}}|\nabla w|^{2} \rightarrow 0 \text { as } n \rightarrow \infty .
$$

Remark 2.1. Note that all integrals are well-defined. Indeed, the first one is defined as $\left|\int_{u_{0}}^{u} h \circ \beta_{0}(r) d r\right| \leq\|h\|_{\infty}\left|u-u_{0}\right|$ and $u \in L^{1}(Q), u_{0} \in L^{1}(\Omega)$. The second integral must be understood as

$$
\int_{\{|w|<k\}}\left(\nabla T_{k} w+F\left(u, T_{k} w\right)\right) \cdot \nabla\left(h\left(T_{k}(w)\right) \xi\right)
$$

for $k>0$ such that Supp $h \subset[-k, k]$. Indeed, if Supp $h \subset[-k, k]$, then $h(w)=h\left(T_{k} w\right)$ and $h(w)=0$ a.e. on $\{|w| \geq k\}$; since $T_{k} w \in L^{2}\left(0, T ; H_{0}^{1}(\Omega)\right)$ it is the same for $h(w) \xi$, and $\nabla(h(w) \xi)=0$ a.e. on $\{|w| \geq k\}$. Similarly the integral (2.4) has to be understood as

$$
\int_{Q \cap\{n \leq|w| \leq n+1\}}\left|\nabla T_{n+1} w\right|^{2} .
$$

The main theorem of this paper is

Theorem 2.1. For any $u_{0} \in L^{1}(\Omega)$ and $g: Q \times \mathbb{R} \rightarrow \mathbb{R}$ satisfying $\left(H_{3}\right)$, there exists a unique renormalized solution $u$ of $E\left(u_{0}, g\right)$. Moreover, $u \in$ $C\left([0, T) ; L^{1}(\Omega)\right), u(0)=u_{0}$, and if $u_{0 i} \in L^{1}(\Omega), g_{i}: Q \times \mathbb{R} \rightarrow \mathbb{R}$ satisfies $\left(H_{3}\right)$ and $u_{i}$ is a renormalized solution of $E\left(u_{0 i}, g_{i}\right)$, for $i=1,2$, then, for all $0 \leq t \leq T$

$$
\int_{\Omega}\left(u_{1}(t)-u_{2}(t)\right)^{+} \leq \int_{\Omega}\left(u_{01}-u_{02}\right)^{+}+\int_{0}^{t} \int_{\Omega} \eta\left(g_{1}\left(., u_{1}\right)-g_{2}\left(., u_{2}\right)\right)
$$

for some $\eta \in \operatorname{sign}^{+}\left(u_{1}-u_{2}\right)$, where $\operatorname{sign}^{+}$denotes the usual non-negative sign graph:

$$
\operatorname{sign}^{+}(s)= \begin{cases}1 & \text { if } s>0 \\ {[0,1]} & \text { if } s=0 \\ 0 & \text { if } s<0\end{cases}
$$




\section{Uniqueness of renormalized solutions}

The proof of Theorem 2.1 will follow as a consequence of Proposition 3.1 below. In fact, we will focus our attention on the problem

$$
\left(E^{\prime}\right)\left(v_{0}, f\right) \begin{cases}\partial_{t} j(v)-\Delta \varphi(v)-\nabla F(j(v), \varphi(v))=f & \text { in } Q \\ \varphi(v)=0 & \text { on } \Sigma \\ j(v)(0, \cdot)=v_{0} & \text { in } \Omega,\end{cases}
$$

where $f \in L^{1}(Q), j, \varphi: \mathbb{R} \rightarrow \mathbb{R}$ are nondecreasing continuous functions such that $j(0)=\varphi(0)=0$, and $v_{0}$ is measurable function such that $u_{0}=j\left(v_{0}\right)$ a.e. on $\Omega$. Indeed, by taking $\varphi=\left(I+\beta^{-1}\right)^{-1}, j=(I+\beta)^{-1}$ and $v:=u+w$, one sees that $E\left(u_{0}, f\right)$ and $E^{\prime}\left(u_{0}, f\right)$ are equivalent.

Remark that

$$
D((j+\varphi))^{-1}=\mathbb{R} .
$$

Next, let us recall the definition of renormalized solution of $E^{\prime}\left(v_{0}, f\right)$.

Definition 3.1. Given $u_{0} \in L^{1}(\Omega)$ and $f \in L^{1}(Q)$, a renormalized solution of $E^{\prime}\left(v_{0}, f\right)$ is a measurable function $v$ such that $u$ is a renormalized solution of $E\left(u_{0}, f\right)$, where $u=j(v)$ and $w=\varphi(v)$.

The main tool we use for the proof of uniqueness of renormalized solution is the following proposition, for which the proof is given at the end of this section.

Proposition 3.1. For any $f_{1}, f_{2} \in L^{1}(Q)$ and $u_{01}, u_{02} \in L^{1}(\Omega)$, if $v_{i}$ is a renormalized solution of $E^{\prime}\left(v_{0 i}, f_{i}\right)$ for $i=1,2$, then, for a.e. $0<t<T$

$$
\begin{aligned}
& \left.\int_{\Omega}\left(\int_{v_{2}(t)}^{v_{1}(t)} h_{n}(\varphi(r)) d j(r)\right)^{+} \leq \int_{\Omega}\left(\int_{v_{02}}^{v_{01}} h_{n}(\varphi(r)) d j(r)\right)\right)^{+} \\
& \quad+\int_{0}^{t} \int_{\Omega} \eta\left(f_{1} h_{n}\left(\varphi\left(v_{1}\right)\right)-f_{2} h_{n}\left(\varphi\left(v_{2}\right)\right)\right) \\
& \quad+\int_{0}^{t} \int_{\Omega} \eta\left(\left(\nabla \varphi\left(v_{1}\right)+F\left(j\left(v_{1}\right), \varphi\left(v_{1}\right)\right)\right) \cdot \nabla h_{n}\left(\varphi\left(v_{1}\right)\right)-\left(\nabla \varphi\left(v_{2}\right)\right.\right. \\
& \left.\left.\quad+F\left(j\left(v_{2}\right), \varphi\left(v_{2}\right)\right)\right) \cdot \nabla h_{n}\left(\varphi\left(v_{2}\right)\right)\right)
\end{aligned}
$$

with $\eta \in \operatorname{Sign}^{+}\left(v_{1}-v_{2}\right)$ a.e. in $Q$.

Corollary 3.1. For any $u_{0} \in L^{1}(\Omega)$ and $g$ satisfying Assumption $\left(H_{3}\right)$ there exists at most one renormalized solution $u$ of $E\left(u_{0}, g\right)$. Moreover, if $u_{0 i} \in$ $L^{1}(\Omega), g_{i}: Q \times \mathbb{R} \rightarrow \mathbb{R}$ satisfies $\left(H_{3}\right)$ and $u_{i}$ is the renormalized solution of $E\left(u_{0 i}, g_{i}\right)$, for $i=1,2$, then (2.5) is fulfilled.

\section{Proof of Theorem 2.1: Uniqueness part.}

First, notice that uniqueness of a renormalized solution follows from (2.5). Indeed, if $u_{01}=u_{02}$ and $g_{1}=g_{2}$, then (2.5) and Assumption $\left(H_{3}\right)$ imply that

$$
\begin{aligned}
\int_{\Omega}\left(u_{1}(t)-u_{2}(t)\right)^{+} & \leq \int_{0}^{t} \int_{\left\{u_{1} \geq u_{2}\right\}}\left(g\left(\cdot, u_{1}\right)-g\left(\cdot, u_{2}\right)\right) \\
& \leq C \int_{0}^{t} \int_{\Omega}\left(u_{1}-u_{2}\right)^{+}
\end{aligned}
$$


which, by Gronwall's Lemma, implies that $u_{1} \leq u_{2}$. In the same way, one can prove that $u_{2} \leq u_{1}$.

Now, let us prove (2.5). It is clear that if $u$ is a renormalized solution of Problem $E\left(u_{0}, g\right)$ with $g$ satisfying Assumption $\left(H_{3}\right)$, then $u$ is a renormalized solution of Problem $E\left(u_{0}, f\right)$ with $f(t, x)=g(t, x, u(t, x))$ a.e. $(t, x) \in Q$. So, it is enough to prove that if $u_{0 i} \in L^{1}(\Omega), f_{i} \in L^{1}(Q)$ and $u_{i}$ is a renormalized solution of $E\left(u_{0 i}, f_{i}\right)$ for $i=1,2$, then, for a.e. $0<t<T$

$$
\int_{\Omega}\left(u_{1}(t)-u_{2}(t)\right)^{+} \leq \int_{\Omega}\left(u_{01}-u_{02}\right)^{+}+\int_{0}^{t} \int_{\Omega}\left(f_{1}-f_{2}\right)^{+},
$$

or, equivalently, for a.e. $0<t<T$

$$
\int_{\Omega}\left(j\left(v_{1}\right)(t)-j\left(v_{2}\right)(t)\right)^{+} \leq \int_{\Omega}\left(j\left(v_{01}\right)-j\left(v_{02}\right)\right)^{+}+\int_{0}^{t} \int_{\Omega}\left(f_{1}-f_{2}\right)^{+},
$$

with $u=j(v)$ and $w=\varphi(v)$, where $v$ is a renormalized solution of $E^{\prime}\left(v_{0}, f\right)$.

To prove the above inequality, we pass to the limit in (3.3) as $n \rightarrow \infty$. So, it is clear that

$$
\begin{aligned}
& \int_{\Omega}\left(\int_{v_{2}(t)}^{v_{1}(t)} h_{n}(\varphi(s)) d j(s)\right)^{+} \rightarrow \int_{\Omega}\left(j\left(v_{1}\right)(t)-j\left(v_{2}\right)(t)\right)^{+}, \\
& \int_{\Omega}\left(\int_{v_{02}}^{v_{01}} h_{n}(\varphi(s) d j(s))^{+} \rightarrow \int_{\Omega}\left(j\left(v_{01}\right)-j\left(v_{02}\right)\right)^{+}\right.
\end{aligned}
$$

and

$$
\int_{0}^{t} \int_{\Omega} \eta\left(f_{1} h_{n}\left(\varphi\left(v_{1}\right)\right)-f_{2} h_{n}\left(\varphi\left(v_{2}\right)\right)\right) \rightarrow \int_{0}^{t} \int_{\Omega} \eta\left(f_{1}-f_{2}\right)^{+} .
$$

The term

$$
\int_{0}^{t} \int_{\Omega} \eta \nabla \varphi\left(v_{1}\right) \cdot \nabla h_{n}\left(\varphi\left(v_{1}\right)\right)-\int_{0}^{t} \int_{\Omega} \eta \nabla \varphi\left(v_{2}\right) \cdot \nabla h_{n}\left(\varphi\left(v_{2}\right)\right)
$$

converges to 0 as $n \rightarrow \infty$ since $\varphi(v)$ satisfies (2.4). Next, let us prove that

$$
\int_{0}^{t} \int_{\Omega} \eta\left(F\left(j\left(v_{1}\right), \varphi\left(v_{1}\right)\right) \cdot \nabla h_{n}\left(\varphi\left(v_{1}\right)\right)-F\left(j\left(v_{2}\right), \varphi\left(v_{2}\right)\right) \cdot \nabla h_{n}\left(\varphi\left(v_{2}\right)\right)\right)=0 .
$$

Define the set

$$
E=\left\{r \in \mathbb{R} ; \varphi_{0}^{-1} \text { is discontinuous at } r\right\}
$$

Since $\varphi_{0}^{-1}$ is a monotone function, $E$ is a countable subset of $\mathbb{R}^{N}$; hence we have

$$
\nabla \varphi(v)=0 \text { a.e. on }\{(t, x) \in Q ; \varphi(v(t, x)) \in E\} \text {. }
$$

From Assumption $\left(H_{2}\right)$, the term $F\left(j\left(v_{1}\right), \varphi\left(v_{1}\right)\right) \cdot \nabla h_{n}\left(\varphi\left(v_{1}\right)\right)$ can be decomposed as

$$
F_{1}\left(\varphi\left(v_{1}\right)\right) \cdot \nabla h_{n}\left(\varphi\left(v_{1}\right)\right)+j\left(v_{1}\right) F_{2}\left(\varphi\left(v_{1}\right)\right) \cdot \nabla h_{n}\left(\varphi\left(v_{1}\right)\right)=: I_{1}+I_{2} .
$$


We have

$$
I_{1}=\int_{Q} \operatorname{div} \int_{0}^{\varphi\left(v_{1}\right)} h_{n}^{\prime}(r) F_{1}(r) d r=0
$$

and

$$
\begin{aligned}
I_{2}= & \int_{E \cap\left\{n<\left|\varphi\left(v_{1}\right)\right|<n+1\right\}} h_{n}^{\prime}\left(\varphi\left(v_{1}\right)\right) j\left(v_{1}\right) F_{2}\left(\varphi\left(v_{1}\right)\right) \cdot \nabla \varphi\left(v_{1}\right) \\
& +\int_{\bar{E} \cap\left\{n<\left|\varphi\left(v_{1}\right)\right|<n+1\right\}} h_{n}^{\prime}\left(\varphi\left(v_{1}\right)\right) j\left(v_{1}\right) F_{2}\left(\varphi\left(v_{1}\right)\right) \cdot \nabla \varphi\left(v_{1}\right) \\
= & I_{2}^{1}+I_{2}^{2},
\end{aligned}
$$

where $\bar{E}$ stands of the complementary of $E$ in $Q$.

From (3.6), $I_{2}^{1}=0$. Since $\varphi^{-1}$ is a continuous function on the set $\bar{E}$, then

$$
I_{2}^{2}=\int_{\bar{E} \cap\left\{n<\left|\varphi\left(v_{1}\right)\right|<n+1\right\}} \operatorname{div} \int_{0}^{\varphi\left(v_{1}\right)} h_{n}^{\prime}(r) j \circ \varphi^{-1}(r) F_{2}(r) d r=0 .
$$

Arguing as above to prove that $\int_{Q} \eta F\left(j\left(v_{2}\right), \varphi\left(v_{2}\right)\right) \cdot \nabla h_{n}\left(\varphi\left(v_{2}\right)\right)=0$.

Finally, collecting all limits, (3.4) follows.

Proof of Proposition 3.1. For any $n \in \mathbb{N}$, let $b_{n}(r)=\int_{0}^{r} h_{n}(\varphi(s)) d j(s)$. Remark that, if $|\varphi(v)| \geq n+1$, then $b_{n}(v)=0$, and if $|\varphi(v)|<n+1$, then the following structure condition holds: if $v<z$

$$
b_{n}(v)=b_{n}(z) \Rightarrow j(v)=j(z) .
$$

By [10], this condition is equivalent to the existence of a continuous function $\widetilde{j}$ such that

$$
j(v)=\widetilde{j}\left(b_{n}(v)\right) .
$$

Let $u_{0} \in L^{1}(\Omega), f \in L^{1}(Q)$ and $v$ a renormalized solution of $E^{\prime}\left(v_{0}, f\right)$ with $v_{0}$ a measurable function such that $u_{0}=j\left(v_{0}\right)$. Hence $v$ satisfies for all $\xi \in$ $\mathcal{D}((0, T) \times \Omega)$

$$
-\int_{Q} \xi_{t} b_{n}(v)+\int_{Q}(\nabla \varphi(v)+F(j(v), \varphi(v))) \cdot \nabla\left(h_{n}(\varphi(v)) \xi\right)=\int_{Q} f h_{n}(v) \xi .
$$

Now, let us consider the second integral in (3.8), which can be written as

$$
\begin{aligned}
\int_{Q}( & \nabla \varphi(v)+F(j(v), \varphi(v))) \cdot \nabla \xi h_{n}(\varphi(v)) \\
& +\int_{Q}(\nabla \varphi(v)+F(j(v), \varphi(v))) \cdot \nabla h_{n}(\varphi(v)) \xi=: K_{1}+K_{2} .
\end{aligned}
$$


From Assumption $\left(H_{2}\right)$ the term $K_{1}$ can be decomposed into three terms $\left(K_{1}^{1}+K_{1}^{2}+K_{1}^{3}\right)$

$$
\begin{aligned}
& \int_{Q} \nabla \varphi(v) \cdot \nabla \xi h_{n}(\varphi(v))+\int_{Q} F_{1}(\varphi(v)) \cdot \nabla \xi h_{n}(\varphi(v)) \\
& \quad+\int_{Q} j(v) F_{2}(\varphi(v)) \cdot \nabla \xi h_{n}(\varphi(v))
\end{aligned}
$$

Note that

$$
K_{1}^{1}=\int_{Q} \nabla H_{n}(\varphi(v)) \cdot \nabla \xi
$$

and

$$
K_{1}^{2}=\int_{Q} \widetilde{F}_{1}\left(H_{n}(\varphi(v))\right) \cdot \nabla \xi
$$

where $\tilde{F}_{1}$ is a continuous function defined by

$$
\widetilde{F}_{1}(r)=h_{n} \circ H_{n}^{-1}(r) F_{1}\left(H_{n}^{-1}(r)\right) .
$$

Since $j(v)=\tilde{j}\left(b_{n}(v)\right)$ on the set where $|\varphi(v)|<n+1$, then

$$
K_{1}^{3}=\int_{Q} \widetilde{j}\left(b_{n}(v)\right) h_{n}(\varphi(v)) F_{2}(\varphi(v)) \cdot \nabla \xi=\int_{Q} \widetilde{j}\left(b_{n}(v)\right) \widetilde{F}_{2}\left(H_{n}(\varphi(v)) \cdot \nabla \xi,\right.
$$

where $\tilde{F}_{2}$ is a continuous function defined by,

$$
\widetilde{F}_{2}(r)=h_{n} \circ H_{n}^{-1}(r) F_{2}\left(H_{n}^{-1}(r)\right) .
$$

Finally, the term $K_{1}^{2}+K_{1}^{3}$ is equal to

$$
\int_{Q} \widetilde{F}\left(b_{n}(v), H_{n}(\varphi(v))\right) \cdot \nabla \xi
$$

where

$$
\widetilde{F}\left(r_{1}, r_{2}\right)=\widetilde{F}_{1}\left(r_{2}\right)+r_{1} \widetilde{F}_{2}\left(r_{2}\right)=h_{n} \circ H_{n}^{-1}\left(r_{2}\right) F\left(\widetilde{j}\left(r_{1}\right), H_{n}^{-1}\left(r_{2}\right)\right) .
$$

Taking account these decompositions, Eq. (3.8) is rewritten as

$$
\begin{gathered}
-\int_{Q} \xi_{t} b_{n}(v)+\int_{Q}\left(\nabla H_{n}(\varphi(v))+\tilde{F}\left(b_{n}(v), H_{n}(\varphi(v))\right)\right) \cdot \nabla \xi \\
=\int_{Q} f h_{n}(\varphi(v)) \xi-\int_{Q}\left(\nabla \varphi(v)+F(j(v), \varphi(v)) \cdot \nabla h_{n}(\varphi(v))\right) \xi
\end{gathered}
$$

which means that $v$ is a weak solution of

$$
\begin{cases}\frac{\partial}{\partial t} b_{n}(v)-\nabla \cdot\left(\nabla H_{n}(\varphi(v))+\tilde{F}\left(b_{n}(v), H_{n}(\varphi(v))\right)\right. & \\ =f h_{n}(\varphi(v))-\nabla \varphi(v)-F(j(v), \varphi(v)) \cdot \nabla h_{n}(\varphi(v)) & \text { in } Q \\ H_{n}(\varphi(v))=0 & \text { on } \Sigma \\ b_{n}(v(0))=b_{n}\left(v_{0}\right) & \text { in } \Omega .\end{cases}
$$


Next, let $u_{i}, i=1,2$ be a renormalized solution of $E\left(u_{0 i}, f_{i}\right)$, then $v_{i}, i=1,2$ is a renormalized solution of $E^{\prime}\left(v_{0 i}, f_{i}\right)$. By the preceding computation, $v_{i}, i=$ 1,2 is also a weak solution of Problem $E_{n}^{\prime}\left(v_{0 i}, f_{i}\right)$, and thanks to [4, Theorem 1], the result of Proposition 3.1 follows.

\section{Existence of weak solutions}

To prove existence of renormalized solutions of Problem $E\left(u_{0}, f\right)$, we will proceed by approximation. We need first to prove, for bounded data $f \in$ $L^{\infty}(Q)$ and $u_{0} \in L^{\infty}(\Omega)$, existence of a weak solution of the parabolic problem with additional strongly monotone perturbation $\psi_{m, n}$, where $\psi_{m, n}(r)=$ $\frac{1}{m} \tan (r)^{+}-\frac{1}{n} \tan (r)^{-}, m, n \in \mathbb{N}:$

$E^{\prime}\left(u_{0}, f, \psi_{m, n}\right) \begin{cases}\partial_{t} j(v)-\Delta \varphi(v)-\nabla F(j(v), \varphi(v))+\psi_{m, n}(v)=f & \text { in } Q \\ \varphi(v)=0 & \text { on } \Sigma \\ j(v)(0, \cdot)=v_{0} & \text { in } \Omega .\end{cases}$

This is done via approximation by a sequence of non-degenerate parabolic problems

$E_{k}^{\prime}\left(v_{0}, f, \psi_{m, n}\right) \begin{cases}\partial_{t} j_{k}(v)-\Delta \varphi_{k}(v)-\nabla F\left(j_{k}(v), \varphi_{k}(v)\right)+\psi_{m, n}(v)=f & \text { in } Q \\ \varphi_{k}(v)=0 & \text { on } \Sigma \\ j_{k}(v)(0, \cdot)=v_{0} & \text { in } \Omega,\end{cases}$

where $j_{k}(r)=j(r)+k r, \varphi_{k}(r)=\varphi(r)+k r\left(\right.$ then $\left.j_{k}^{-1}, \varphi_{k}^{-1} \in C_{0}(\mathbb{R})\right)$.

For these non-degenerate problems we obtain existence of weak solutions with appropriate estimates and monotonicity properties, which allow us to pass to the limit.

So, let us define the operator $A_{m, n}$, in $L^{1}(\Omega)$, by

$$
A_{m, n}(z)=-\Delta \varphi(z)-\nabla F(j(z), \varphi(z))+\psi_{m, n}(\varphi(z)) \text { in } \mathcal{D}^{\prime}(\Omega)
$$

and

$$
\mathcal{D}\left(A_{m, n}\right)=\left\{z \in L^{\infty}(\Omega) ; \varphi(z) \in H_{0}^{1}(\Omega), A_{m, n}(z) \in L^{1}(\Omega)\right\} .
$$

Thanks to the results of [15], we know that $A_{m, n}$ is $T$-accretive in $L^{1}(\Omega)$, and $\overline{A_{m, n}}$, the closure of $A_{m, n}$ in $L^{1}$, is $m$-accretive in $L^{1}(\Omega)$, and moreover $\overline{D\left(A_{m, n}\right)}=L^{1}(\Omega)$.

Moreover, if $\left(j_{k}\right)_{k},\left(\varphi_{k}\right)_{k}$ are continuous and nondecreasing functions with $j_{k}(0)=\varphi_{k}(0)=0$ such that $j_{k} \rightarrow j$ and $\varphi_{k} \rightarrow \varphi$ uniformly, then $A_{m, n} \subseteq$ $\liminf _{k \rightarrow 0} A_{m, n}^{k}$, where the operator $A_{m, n}^{k}$ is defined as $A_{m, n}$, by replacing $j$ and $\varphi$ by $j_{k}$ and $\varphi_{k}$ respectively.

According to these results, by nonlinear semigroups theory, for any $k, m$, $n \in \mathbb{N}$, for all $v_{0} \in L^{1}(\Omega)$, for all $f \in L^{1}(Q)$, there exits a unique mild solution $v_{m, n}^{k} \in C\left([0, T] ; L^{1}(\Omega)\right)$ of the abstract Cauchy problem in $L^{1}(\Omega)$

$$
\frac{d v}{d t}+A_{m, n}^{k} v \ni f, v(0)=v_{0}
$$


Moreover, for any $v_{0}^{k} \in L^{1}(\Omega)$ with $v_{0}^{k} \rightarrow v_{0}$ in $L^{1}(\Omega)$ and for all $f \in L^{1}(Q)$, the mild solution $v_{m, n}^{k}$ of (4.11) with initial data $v_{0}^{k}$ converges in $C\left([0, T] ; L^{1}(\Omega)\right)$ as $k \rightarrow 0$ to the mild solution $v_{m, n}$ of the Cauchy problem

$$
\frac{d v}{d t}+A_{m, n} v \ni f, v(0)=v_{0} .
$$

For bounded data, we can prove that the mild solution of the Cauchy problem (4.12) is a "weak solution".

Definition 4.1. A weak solution of $E\left(u_{0}, f\right)$ is a couple of functions $(u, w)$ such that $u \in L^{\infty}(Q), w \in L^{2}\left(0, T ; H_{0}^{1}(\Omega)\right), w \in \beta(u), F(u, w) \in\left(L^{2}(Q)\right)^{N}$, and

$$
\int_{Q}\left[(\nabla w+F(u, w)) \cdot \nabla \xi-u \xi_{t}\right]=\int_{Q} f \xi-\int_{\Omega} \xi(0) u_{0}
$$

for all $\xi \in \mathcal{D}((-\infty, T) \times \Omega)$.

Next, let us recall the definition of weak solution of $E^{\prime}\left(v_{0}, f\right)$.

Definition 4.2. Given $u_{0} \in L^{\infty}(\Omega)$ and $f \in L^{\infty}(Q)$, a weak solution of $E^{\prime}\left(v_{0}, f\right)$ is a measurable function $v$ such that the couple $(u, w)$ is a weak solution of $E\left(u_{0}, f\right)$, where $u=j(v)$ and $w=\varphi(v)$.

It is proved by Carrillo [15] the following result:

Proposition 4.1. [15] Let $m, n, k \in \mathbb{N}$, for $f \in L^{\infty}(Q)$ and $u_{0} \in L^{\infty}(\Omega)$ let $v_{m, n}^{k}$ be the mild solution of (4.11). Then $v_{m, n}^{k}$ is a weak solution of $E_{k}^{\prime}\left(v_{0}, f, \psi_{m, n}\right)$.

Proposition 4.2. Given $u_{0} \in L^{\infty}(\Omega)$ and $f \in L^{\infty}(Q)$ there exists a weak solution of Problem $E^{\prime}\left(v_{0}, f, \psi_{m, n}\right)$.

Proof of Proposition 4.2. From now on and until Sect. 5, we omit the index $m, n$ to lighten the notations. by [15]

Recall that $v_{k}$ is the mild solution of $\frac{d v_{k}}{d t}+A_{m, n}^{k} v_{k} \ni f, v_{k}(0)=v_{0}^{k}$, thus,

$$
\left\|j_{k}\left(v_{k}\right)\right\|_{\infty} \leq C\left(f, v_{0}, m, n\right) .
$$

Let $B_{k}(s)=\int_{0}^{s} \varphi_{k} \circ j_{k}^{-1}(r) d r$. By taking $\xi=\varphi_{k}\left(v_{k}\right)$ as a test function in the weak formulation of the solution $v_{k}$, and by using Lemma 2.1, yields

$$
\begin{aligned}
\int_{\Omega} & B_{k}\left(v_{k}\right)+\int_{Q}\left(\nabla \varphi_{k}\left(v_{k}\right)+F\left(j_{k}\left(v_{k}\right), \varphi_{k}\left(v_{k}\right)\right)\right) \cdot \nabla \varphi_{k}\left(v_{k}\right) \\
& +\int_{Q} \psi_{m, n}\left(\varphi_{k}\left(v_{k}\right)\right) \varphi_{k}\left(v_{k}\right) \\
= & \int_{Q} f \varphi_{k}\left(v_{k}\right)+\int_{\Omega} B_{k}\left(v_{k}^{0}\right) .
\end{aligned}
$$

By monotonicity of $\psi_{m, n}, \int_{Q} \psi_{m, n}\left(\varphi_{k}\left(v_{k}\right)\right) \varphi_{k}\left(v_{k}\right) \geq 0$. The term in the second integral on the right hand side $\left.F\left(j_{k}\left(v_{k}\right), \varphi_{k}\left(v_{k}\right)\right)\right) \cdot \nabla \varphi_{k}\left(v_{k}\right)$; from Assumption $\left(\mathrm{H}_{2}\right)$ we have 


$$
\begin{aligned}
F\left(j_{k}\left(v_{k}\right), \varphi_{k}\left(v_{k}\right)\right) \cdot \nabla \varphi_{k}\left(v_{k}\right)= & F_{1}\left(\varphi_{k}\left(v_{k}\right)\right) \cdot \nabla \varphi_{k}\left(v_{k}\right) \\
& +j_{k}\left(v_{k}\right) F_{2}\left(\varphi_{k}\left(v_{k}\right)\right) \cdot \nabla \varphi_{k}\left(v_{k}\right),
\end{aligned}
$$

whence

$$
\int_{Q} F_{1}\left(\varphi_{k}\left(v_{k}\right)\right) \cdot \nabla \varphi_{k}\left(v_{k}\right)=\int_{Q} \operatorname{div} \int_{0}^{\varphi_{k}\left(v_{k}\right)} F_{1}(r) d r=0 ;
$$

and, since $\varphi_{k}^{-1}$ is a continuous function almost everywhere in $\Omega$, we get

$$
\begin{aligned}
\int_{Q} j_{k}\left(v_{k}\right) F_{2}\left(\varphi_{k}\left(v_{k}\right)\right) \cdot \nabla \varphi_{k}\left(v_{k}\right) & =\int_{Q} j_{k} \circ \varphi_{k}^{-1}\left(\varphi_{k}\left(v_{k}\right)\right) F_{2}\left(\varphi_{k}\left(v_{k}\right)\right) \cdot \nabla \varphi_{k}\left(v_{k}\right) \\
& =\int_{Q} \operatorname{div} \int_{0}^{\varphi_{k}\left(v_{k}\right)} j_{k} \circ \varphi_{k}^{-1}(r) F_{2}(r) d r=0 .
\end{aligned}
$$

Then, we get from (4.13) that $\varphi_{k}\left(v_{k}\right)$ is bounded in $L^{2}\left(0, T ; H_{0}^{1}(\Omega)\right)$, hence, there exists a subsequence, still denoted by $k$, such that

$$
\varphi_{k}\left(v_{k}\right) \rightarrow w \text { weakly in } L^{2}\left(0, T ; H_{0}^{1}(\Omega)\right) .
$$

One can prove exactly as $[3,10]$ that $\varphi_{k}\left(v_{k}\right)$ is uniformly bounded in $L^{\infty}(Q)$.

It remains to prove the strong convergence of $\varphi_{k}\left(v_{k}\right)$ in $L^{1}(Q)$.

The proof is based on Kruzhkov's method of doubling of variables. Let $t, s \in[0, T], k, l \in \mathbb{N}$, and consider the weak solution $v_{k}(t, x)$ as a function of $(t, x)$ and $v_{l}(s, x)$ as a function of $(s, x)$. Choose in each weak formulation the test function $\phi=\frac{1}{h} \int_{t}^{t+h} \eta_{\delta}\left(\varphi_{k}\left(v_{k}\right)-\varphi_{l}\left(v_{l}\right)+\delta \zeta\right) \xi$, where $\xi \in C_{c}^{\infty}\left([0, T)^{2} \times\right.$ $\bar{\Omega}), \xi \geq 0, \zeta \in C_{c}^{\infty}(\Omega), 0 \leq \zeta \leq 1$ and $\eta_{\delta}(r)=\frac{T_{\delta}(r)}{\delta}$, and integrate in $t$. Using Lemma 2.1 in each inequality, taking their difference, passing to the limit with $h \rightarrow 0$ exactly as in [28, Proposition 4.2.2] (see also [12, Proposition 4.2]) yields

$$
\begin{aligned}
& -\int_{0}^{T} \int_{Q} \xi_{t}\left[\left|j_{k}\left(v_{k}\right)-j_{l}\left(v_{l}\right)\right|-\left|v_{k}^{0}-j_{l}\left(v_{l}\right)\right|\right] \\
& -\int_{0}^{T} \int_{Q} \xi_{s}\left[\left|j_{k}\left(v_{k}\right)-j_{l}\left(v_{l}\right)\right|-\left|v_{l}^{0}-j_{k}\left(v_{k}\right)\right|\right] \\
& +\int_{0}^{T} \int_{Q}\left(\chi_{\left\{\varphi_{k}\left(v_{k}\right)>\varphi_{l}\left(v_{l}\right)\right\}}-\chi_{\left\{\varphi_{k}\left(v_{k}\right)<\varphi_{l}\left(v_{l}\right)\right\}}\right)\left(\nabla\left(\varphi_{k}\left(v_{k}\right)-\varphi_{l}\left(v_{l}\right)\right)\right) \cdot \nabla \xi \\
& +\int_{0}^{T} \int_{Q}\left(\chi_{\left\{\varphi_{k}\left(v_{k}\right)>\varphi_{l}\left(v_{l}\right)\right\}}-\chi_{\left\{\varphi_{k}\left(v_{k}\right)<\varphi_{l}\left(v_{l}\right)\right\}}\right)\left(F\left(j_{k}\left(v_{k}\right), \varphi_{k}\left(v_{k}\right)\right)\right. \\
& \left.-F\left(j_{l}\left(v_{k}\right), \varphi_{l}\left(v_{l}\right)\right)\right) \cdot \nabla \xi \\
& +\int_{0}^{T} \int_{Q}\left(\psi_{m, n}\left(\varphi_{k}\left(v_{k}\right)\right)-\psi_{m, n}\left(\varphi_{l}\left(v_{l}\right)\right)\right)\left(\chi_{\left\{\varphi_{k}\left(v_{k}\right)>\varphi_{l}\left(v_{l}\right)\right\}}\right. \\
& \left.-\chi_{\left\{\varphi_{k}\left(v_{k}\right)<\varphi_{l}\left(v_{l}\right)\right\}}\right) \xi \\
& \leq \int_{0}^{T} \int_{Q}(f(t, x)-f(s, x))\left(\chi_{\left\{\varphi_{k}\left(v_{k}\right)>\varphi_{l}\left(v_{l}\right)\right\}}-\chi_{\left\{\varphi_{k}\left(v_{k}\right)<\varphi_{l}\left(v_{l}\right)\right\}}\right. \\
& \left.+\chi_{\left\{\varphi_{k}\left(v_{k}\right)=\varphi_{l}\left(v_{l}\right)\right\}}\right) \xi
\end{aligned}
$$


The proof of the above inequality is given in $[28,29]$. The original proof can be found in [12]. We omit here the details in order to avoid the unnecessary duplication of arguments.

Take $\xi=\phi(t) \rho_{p}(t-s)$, with $\phi \in C_{c}^{\infty}([0, T)), \phi \geq 0$ and $\left(\rho_{p}\right)_{p}$ be a classical sequence of mollifiers in $\mathbb{R}$ with $\operatorname{Supp}\left(\rho_{p}\right) \subset\left[-\frac{2}{p}, 0\right]$. Pass to the limit with $p \rightarrow 0$, yields

$$
\lim _{p \rightarrow 0} \lim _{k, l \rightarrow 0} \int_{0}^{T} \int_{Q} \phi \rho_{p}\left|\psi_{m, n}\left(\varphi_{k}\left(v_{k}(t, x)\right)\right)-\psi_{m, n}\left(\varphi_{l}\left(v_{l}(s, x)\right)\right)\right| \leq 0 .
$$

In the particular case $k=l$, the preceding arguments lead to the estimate

$$
\lim _{p \rightarrow 0} \lim _{l \rightarrow 0} \int_{0}^{T} \int_{Q}\left|\psi_{m, n}\left(\varphi_{l}\left(v_{l}(t, x)\right)\right)-\psi_{m, n}\left(\varphi_{l}\left(v_{l}(s, x)\right)\right)\right| \phi \rho_{p} \leq 0 .
$$

By choosing $\phi$ such that $\phi=1$ on $[\tau, \theta]$, where $0<\tau<\theta<T$, we get

$$
\begin{aligned}
& \lim _{k, l \rightarrow 0} \int_{\tau}^{\theta} \int_{\Omega}\left|\psi_{m, n}\left(\varphi_{k}\left(v_{k}(t, x)\right)\right)-\psi_{m, n}\left(\varphi\left(v_{l}(t, x)\right)\right)\right| \\
& \leq \lim _{p \rightarrow \infty} \lim _{k, l \rightarrow 0} \int_{0}^{T} \int_{Q}\left|\psi_{m, n}\left(\varphi_{k}\left(v_{k}(t, x)\right)\right)-\psi_{m, n}\left(\varphi_{l}\left(v_{l}(t, x)\right)\right)\right| \phi \rho_{p} \\
& \leq \lim _{p \rightarrow \infty} \lim _{k, l \rightarrow 0}\left(\int_{0}^{T} \int_{Q}\left|\psi_{m, n}\left(\varphi_{k}\left(v_{k}(t, x)\right)\right)-\psi_{m, n}\left(\varphi_{l}\left(v_{l}(s, x)\right)\right)\right| \phi \rho_{p}\right. \\
& \left.\quad+\int_{0}^{T} \int_{Q}\left|\psi_{m, n}\left(\varphi_{l}\left(v_{l}(s, x)\right)\right)-\psi_{m, n}\left(\varphi_{l}\left(v_{l}(t, x)\right)\right)\right| \phi \rho_{p}\right) \\
& \leq 0 .
\end{aligned}
$$

As $\psi_{m, n}$ is strictly nondecreasing, it follows that

$$
\lim _{k, l \rightarrow 0} \int_{\tau}^{\theta} \int_{\Omega}\left|\varphi_{k}\left(v_{k}\right)-\varphi_{l}\left(v_{l}\right)\right|=0 \quad \forall 0<\tau<\theta<T .
$$

Since $\left(\varphi_{k}\left(v_{k}\right)\right)_{k}$ is bounded in $L^{\infty}(Q)$ and $\varphi_{k}\left(v_{k}\right) \rightarrow w$ weakly in $L^{2}\left(0, T ; H_{0}^{1}\right.$ $(\Omega)$ ), we conclude that

$$
\varphi_{k}\left(v_{k}\right) \rightarrow w \text { strongly in } L^{1}(Q) \text {, and a.e. on } Q \text {. }
$$

By nonlinear semigroup theory, $j_{k}\left(v_{k}\right) \rightarrow u$ in $L^{\infty}\left(0, T ; L^{1}(\Omega)\right)$, we deduce existence of subsequence of $k$, still denoted by $k$, such that

$$
j_{k}\left(v_{k}\right) \rightarrow u \text { a.e. on } Q \text {. }
$$

The task now is to prove that

$$
u=j(v) \text { and } w=\varphi(v) .
$$

Since $\varphi=\varphi_{k}-k I, j=j_{k}-k I$, and $\varphi_{k}\left(v_{k}\right), j_{k}\left(v_{k}\right)$ are uniformly bounded in $L^{\infty}(Q)$, then, almost everywhere on $Q$, we have

$$
\left|j\left(v_{k}\right)+\varphi\left(v_{k}\right)\right| \leq\left|j_{k}\left(v_{k}\right)+\varphi_{k}\left(v_{k}\right)\right| \leq C .
$$

From (3.2) we deduce the existence of a constant $C$ such that

$$
\left\|v_{k}\right\|_{L^{\infty}(Q)} \leq C .
$$


Moreover, $v_{k}$ converges to $v^{*}$-weakly in $L^{\infty}(Q)$, still converges in $L^{2}(Q)$, and $k v_{k}$ converges to 0 in $L^{\infty}(Q)$.

We deduce also that $\varphi\left(v_{k}\right)=\varphi_{k}\left(v_{k}\right)-k v_{k}$ still converges to $w$ in $L^{2}(Q)$, whence we deduce that $w=\varphi(v)$. Also we have $u=j(v)$.

Therefore, since $j_{k}\left(v_{k}\right), \varphi_{k}\left(v_{k}\right)$ are uniformly bounded in $L^{\infty}(Q)$, we have $F\left(j_{k}\left(v_{k}\right), \varphi_{k}\left(v_{k}\right)\right)$ is uniformly bounded in $\left(L^{\infty}(Q)\right)^{N}$ since $F_{1}, F_{2}$ are continuous. Hence, from Lebesgue Theorem, we deduce that

$$
F\left(j_{k}\left(v_{k}\right), \varphi_{k}\left(v_{k}\right)\right) \rightarrow F(j(v), \varphi(v)) \text { in } L^{1}(Q) .
$$

Now, let $\xi \in C_{c}^{\infty}([0, T) \times \Omega)$, then

$$
\begin{aligned}
\int_{Q} & -j_{k}\left(v_{k}\right) \xi_{t}+\int_{Q}\left(\nabla \varphi_{k}\left(v_{k}\right)+F\left(j_{k}\left(v_{k}\right), \varphi_{k}\left(v_{k}\right)\right)\right) \cdot \nabla \xi+\int_{Q} \psi_{m, n}\left(\varphi_{k}\left(v_{k}\right)\right) \xi \\
= & \int_{Q} f \xi-\int_{\Omega} v_{k}^{0} \xi(0),
\end{aligned}
$$

and by letting $k \rightarrow 0$ we get

$$
\begin{aligned}
\int_{Q} & -j(v) \xi_{t}+\int_{Q}(\nabla \varphi(v)+F(j(v), \varphi(v))) \cdot \nabla \xi+\int_{Q} \psi_{m, n}(\varphi(v)) \xi \\
= & \int_{Q} f \xi-\int_{\Omega} v_{0} \xi(0) .
\end{aligned}
$$

Hence $v$ is a weak solution of $E^{\prime}\left(v_{0}, f, \psi_{m, n}\right)$. Consequently $u$ is a weak solution of $E\left(u_{0}, f, \psi_{m, n}\right)$ with $u=j(v)$ and $w=\varphi(v)$.

\section{Existence of renormalized solutions}

The main result of this section is

Theorem 5.1. For all $u_{0} \in L^{1}(\Omega)$ and $f \in L^{1}(Q)$ Problem $E\left(u_{0}, f\right)$ admits a renormalized solution.

Proof. Following a standard approach, we obtain the existence of a solution as limit of approximating problems. To this purpose let $u_{m, n}^{0}=\sup \left\{\inf \left\{m, u_{0}\right\}\right.$, $-n\} \in L^{\infty}(\Omega)$, and $f_{m, n}=\sup \{\inf \{m, f\},-n\} \in L^{\infty}(Q)$ be a bi-monotone approximation of $u_{0}$ and $f$ in $L^{1}$. Then, by Proposition 4.2 , there exists a weak solution $u_{m, n}$ of Problem $E\left(u_{m, n}^{0}, f_{m, n}, \psi_{m, n}\right)$, i.e.

$$
\begin{aligned}
& u_{m, n_{t}}-\Delta w_{m, n}-\nabla F\left(u_{m, n}, w_{m, n}\right)+\psi_{m, n}\left(w_{m, n}\right)=f_{m, n}, \\
& w_{m, n} \in \beta\left(u_{m, n}\right) \text { in } \mathcal{D}^{\prime}(Q),
\end{aligned}
$$

which is equivalent to

$$
\begin{aligned}
& j\left(v_{m, n}\right)_{t}-\Delta \varphi\left(v_{m, n}\right)-\nabla F\left(j\left(v_{m, n}\right), \varphi\left(v_{m, n}\right)\right)+\psi_{m, n}\left(\varphi\left(v_{m, n}\right)\right) \\
& \quad=f_{m, n} \text { in } \mathcal{D}^{\prime}(Q)
\end{aligned}
$$

with $u_{m, n}=j\left(v_{m, n}\right)$ and $w_{m, n}=\varphi\left(u_{m, n}\right)$.

We are going to prove that the limit a.e. of $u_{m, n}$, respectively of $j\left(v_{m, n}\right)$, is a renormalized solution of $E\left(u_{0}, f\right)$, respectively of $E^{\prime}\left(v_{0}, f\right)$. 
By choosing in (5.15) the test function $T_{k}\left(\varphi\left(v_{m, n}\right)\right)$ and using Lemma 2.1 , yields

$$
\begin{aligned}
\int_{Q} & \left|\nabla T_{k}\left(\varphi\left(u_{m, n}\right)\right)\right|^{2}+\int_{\left\{\left|\varphi\left(u_{m, n}\right)\right| \leq k\right\}} F\left(j\left(v_{m, n}\right), \varphi\left(v_{m, n}\right)\right) \cdot \nabla \varphi\left(v_{m, n}\right) \\
& +\int_{Q} \psi_{m, n}\left(\varphi\left(v_{m, n}\right)\right) T_{k}\left(\varphi\left(u_{m, n}\right)\right) \\
\leq & k\left(\int_{Q}\left|f_{m, n}\right|+\int_{\Omega}\left|j\left(v_{m, n}^{0}\right)\right|\right) .
\end{aligned}
$$

As in (3.5), it follows that $\int_{\left\{\mid \varphi\left(u_{m, n} \mid \leq k\right\}\right.} F\left(j\left(v_{m, n}\right), \varphi\left(v_{m, n}\right)\right) \cdot \nabla \varphi\left(v_{m, n}\right)=0$. By monotonicity of the function $\psi_{m, n}$ we deduce from inequality (5.16)

$$
\int_{Q}\left|\nabla T_{k} \varphi\left(u_{m, n}\right)\right|^{2} \leq k C,
$$

where $C$ is a constant independent of $m, n$. Thus $T_{k} \varphi\left(u_{m, n}\right)$ is bounded in $L^{2}\left(0, T ; H_{0}^{1}(\Omega)\right)$. Hence, up to a subsequence,

$$
T_{k} \varphi\left(u_{m, n}\right) \rightarrow g \text { weakly in } L^{2}\left(0, T ; H_{0}^{1}(\Omega)\right) \text { as } m, n \rightarrow \infty .
$$

Now let us prove, up to a subsequence, the strong convergence of the sequence $\left(\varphi\left(v_{m, n}\right)\right)_{m, n}$. For this we will use the following comparison result

Lemma 5.1. Let $v_{0}, \tilde{v}_{0} \in L^{\infty}(\Omega), f, \tilde{f} \in L^{\infty}(Q), \psi, \tilde{\psi}: \mathbb{R} \rightarrow \mathbb{R}$ continuous, strictly increasing functions with $\psi(0)=\tilde{\psi}(0)=0$, and let $v, \tilde{v}$ be weak solutions of $E^{\prime}\left(v_{0}, f, \psi\right), E^{\prime}\left(\tilde{v}_{0}, \tilde{f}, \tilde{\psi}\right)$ respectively. Then

$\int_{\Omega}(j(v)(t)-j(\tilde{v}))^{+}+\int_{Q}(\psi(\varphi(v))-\tilde{\psi}(\varphi(\tilde{v})))^{+} \leq \int_{Q}(f-\tilde{f})^{+}-\int_{\Omega}\left(v_{0}-\tilde{v}_{0}\right)^{+}$.

Proof. The proof is adapted exactly from the proof of inequality (4.14). It suffices to take in the equations corresponding to the weak solutions $v$ and $\tilde{v}$ the test functions $\frac{1}{h} \int_{t}^{t+h} \eta_{\delta}^{+}(\varphi(v)-\varphi(\tilde{v})+\delta \zeta)$, where $\eta_{\delta}^{+}(r)=\frac{T_{\delta}^{+}(r)}{\delta}$.

From Lemma 5.1, we obtain, for $v_{m, n}$ weak solution of $E^{\prime}\left(v_{m, n}^{0}, f_{m, n}, \psi_{m, n}\right)$,

$$
\int_{Q}\left(\psi_{m, n}\left(\varphi\left(v_{m, n}\right)\right)-\psi_{m+1, n}\left(\varphi\left(v_{m+1, n}\right)\right)\right)^{+} \leq 0 .
$$

Since $\psi_{m+1, n}(r) \leq \psi_{m, n}(r)$ and $\psi_{m+1, n}$ is strictly increasing, then for all $m, n>0$

$$
\varphi\left(v_{m, n}\right) \leq \varphi\left(v_{m+1, n}\right) \text { a.e. on } Q .
$$

The same reasoning implies that for all $m, n>0 \quad \varphi\left(v_{m, n}\right) \geq \varphi\left(v_{m, n+1}\right)$ a.e. on $Q$. Therefore, thanks to the monotone convergence theorem

$$
\varphi\left(v_{m, n}\right) \uparrow_{m} w_{n} \downarrow_{n} w \text { in } L^{1}(Q),
$$

where $w_{n}, w: Q \rightarrow \mathbb{R}$ are measurable functions, finite a.e. on $Q$. Here and in the sequel, we use the notation $\uparrow_{m}$ respectively $\downarrow_{m}$, to denote convergence of a sequence which is monotone increasing, respectively decreasing, in $m$.

Applying the diagonal procedure, we may assume that, for some sequence $m(n), \varphi\left(v_{n}\right):=\varphi\left(v_{m(n), n}\right) \rightarrow w$ in $L^{1}(Q)$. 
Extracting a subsequence if necessary, we may therefore assume that

$$
T_{k} \varphi\left(v_{n}\right) \rightarrow T_{k} w \text { weakly in } L^{2}\left(0, T ; H_{0}^{1}(\Omega)\right) \text { for all } k>0
$$

and

$$
\varphi\left(v_{n}\right) \rightarrow w \text { a.e. on } Q \text {. }
$$

As $v_{n}$ is a mild solution of $\frac{d v}{d t}+A_{m(n), n} v \ni f, v(0)=v_{0}$

$$
u_{n}:=j\left(v_{n}\right) \rightarrow u \text { in } L^{\infty}\left(0, T ; L^{1}(\Omega)\right) .
$$

Since $\varphi\left(v_{n}\right)$ converges weakly in $L^{2}(Q)$, and since $\varphi \circ j^{-1}$ is a maximal monotone operator (in $\left.L^{2}(Q)\right)$, we deduce that

$$
w \in \varphi \circ j^{-1}(u),
$$

whence there exits $\tilde{u} \in j^{-1}(u)$ such that $w=\varphi(\tilde{u})$. Then we set

$$
v=\left((\varphi+j)^{-1}\right)_{0}(u+w)=\left((\varphi+j)^{-1}\right)_{0}(\varphi(\tilde{u})+j(\tilde{u})) .
$$

Obviously, $v$ is a measurable function and we have $u=j(v)$ and $w=\varphi(v)$.

We may assume that for some sequence $(m(n))_{n}$, we have (with $f_{n}=f_{m(n), n}$, $\left.v_{n}^{0}=v_{m(n), n}^{0}, \psi_{n}=\psi_{m(n), n}\right)$

$$
\begin{aligned}
& f_{n} \rightarrow f \text { in } L^{1}(Q), \\
& j\left(v_{n}^{0}\right) \rightarrow u_{0} \quad \text { in } L^{1}(\Omega)
\end{aligned}
$$

and the weak solution $v_{n}$ of $E^{\prime}\left(v_{n}^{0}, f_{n}, \psi_{n}\right)$ satisfies

$$
\varphi\left(v_{n}\right) \rightarrow \varphi(v) \text { a.e. on } Q
$$

and

$$
j\left(v_{n}\right) \rightarrow u \text { in } L^{\infty}\left(0, T ; L^{1}(\Omega)\right), \text { a.e. on } Q .
$$

The task now is to prove that

$$
\left|\nabla T_{k} \varphi\left(v_{n}\right)\right|^{2} \rightarrow\left|\nabla T_{k} \varphi(v)\right|^{2} \text { in } L^{1}(Q) \text { as } n \rightarrow \infty .
$$

For this we need to recall the following definition of a time regularization of $T_{k}(u)$, which was first introduced in [24], and used in several papers afterward (see e.g. $[2,3,5,6,12])$. Let $\nu>0$ and $\left(w_{\nu}^{0}\right)_{\nu}$ be a sequence of functions such that

$$
\left\{\begin{array}{l}
w_{\nu}^{0} \in H_{0}^{1}(\Omega) \cap L^{\infty}(\Omega) \\
\left\|w_{\nu}^{0}\right\|_{L^{\infty}(\Omega)} \leq k \\
w_{\nu}^{0} \rightarrow T_{k} \varphi(v(0)) \text { a.e. on } \Omega \text { as } \nu \rightarrow \infty \\
\frac{1}{\nu}\left\|w_{\nu}^{0}\right\|_{H_{0}^{1}(\Omega)} \rightarrow 0 \text { as } \nu \rightarrow \infty
\end{array}\right.
$$

Then, for all $k, \nu>0$, we denote by $\left(T_{k} \varphi(v)\right)_{\nu}$ the unique solution of the problem

$$
\begin{cases}\frac{\partial\left(T_{k} \varphi(v)\right)_{\nu}}{\partial t}=\nu\left(T_{k} \varphi(v)-\left(T_{k} \varphi(v)\right)_{\nu}\right) & \text { on } Q \\ \left(T_{k} \varphi(v)\right)_{\nu}(0, \cdot)=w_{\nu}^{0} & \text { on } \Omega\end{cases}
$$


Then $\left(T_{k} \varphi(v)\right)_{\nu} \in L^{2}\left(0, T ; H_{0}^{1}(\Omega)\right) \cap L^{\infty}(Q), \frac{\partial\left(T_{k} \varphi(v)\right)_{\nu}}{\partial t} \in L^{2}\left(0, T ; H_{0}^{1}(\Omega)\right) \cap$ $L^{\infty}(Q)$, and up to a subsequence, we can assume that

$$
\begin{aligned}
& \left(T_{k} \varphi(v)\right)_{\nu} \rightarrow T_{k} \varphi(v) \text { strongly in } L^{2}\left(0, T ; H_{0}^{1}(\Omega)\right), \\
& \left(T_{k} \varphi(v)\right)_{\nu}(t) \rightarrow T_{k} \varphi(v)(t) \text { a.e. on } \Omega \text { for a.e. } t
\end{aligned}
$$

and

$$
\left\|\left(T_{k} \varphi(v)\right)_{\nu}\right\|_{L^{\infty}(Q)} \leq k \quad \forall \nu>0 .
$$

Let $\sigma \in \mathcal{D}_{+}(0, T)$ and $h_{l}(r)=(l+1-|r|)^{+} \wedge 1, l \in \mathbb{N}, l>k$. We prove that, for any fixed $k>0$,

$$
\liminf _{l \rightarrow \infty} \liminf _{\nu \rightarrow \infty} \lim _{n \rightarrow \infty} \int_{Q} \sigma \nabla \varphi\left(v_{n}\right) \cdot \nabla\left(h_{l}\left(\varphi\left(u_{n}\right)\right)\left(T_{k} \varphi\left(v_{n}\right)-\left(T_{k} \varphi(v)\right)_{\nu}\right)\right) \leq 0 .
$$

To this end, consider $\left.\sigma h_{l}\left(u_{m, n}\right)\left(T_{k} \varphi\left(v_{n}\right)\right)-\left(T_{k} \varphi(v)\right)_{\nu}\right)$ as a test function in (5.15) and pass to the limit with $n \rightarrow \infty$ in each term. We use the same techniques as in [3, Proof of Theorem 2.4] to prove that

$$
\liminf _{\nu \rightarrow \infty} \lim _{n \rightarrow \infty}\left\langle j\left(v_{n}\right)_{t}, \sigma h_{l}\left(\varphi\left(v_{n}\right)\right)\left(T_{k} \varphi\left(v_{n}\right)-\left(T_{k} \varphi(v)\right)_{\nu}\right)\right\rangle \geq 0,
$$

where $\langle\cdot, \cdot\rangle$ denotes the paring between $L^{2}\left(0, T ; H_{0}^{1}(\Omega)\right)$ and $L^{2}\left(0, T ; H^{-1}(\Omega)\right)$. As in (3.5), we show that

$$
\lim _{\nu \rightarrow \infty} \lim _{n \rightarrow \infty} \int_{Q} \sigma F\left(j\left(v_{n}\right), \varphi\left(v_{n}\right)\right) \cdot \nabla\left(h_{l}\left(\varphi\left(v_{n}\right)\right)\left(T_{k} \varphi\left(v_{n}\right)-\left(T_{k} \varphi(v)\right)_{\nu}\right)\right)=0 .
$$

It is clear that

$$
\lim _{\nu \rightarrow \infty} \lim _{n \rightarrow \infty} \int_{Q} \psi_{n}\left(\varphi\left(v_{n}\right)\right) \sigma h_{l}\left(\varphi\left(v_{n}\right)\right)\left(T_{k} \varphi\left(v_{n}\right)-\left(T_{k} \varphi(v)\right)_{\nu}\right)=0
$$

and

$$
\lim _{\nu \rightarrow \infty} \lim _{n \rightarrow \infty} \int_{Q} f_{n} \sigma h_{l}\left(\varphi\left(v_{n}\right)\right)\left(T_{k}\left(\varphi\left(v_{n}\right)\right)-\left(T_{k} \varphi(v)\right)_{\nu}\right)=0 .
$$

An equivalent formulation of (5.21) is

$$
\begin{aligned}
& \limsup _{\nu \rightarrow \infty} \limsup _{n \rightarrow \infty}\left(\int_{Q} \sigma h_{l}\left(\varphi\left(v_{n}\right)\right) \nabla \varphi\left(v_{n}\right) \cdot \nabla\left(T_{k} \varphi\left(v_{n}\right)-\left(T_{k} \varphi(v)\right)_{\nu}\right)\right. \\
& \left.\quad+\int_{\left\{l<\left|\varphi\left(v_{n}\right)\right|<l+1\right\}} \sigma h_{l}^{\prime}\left(\varphi\left(v_{n}\right)\right)\left(T_{k} \varphi\left(v_{n}\right)-\left(T_{k} \varphi(v)\right)_{\nu}\right) \nabla \varphi\left(v_{n}\right) \cdot \nabla \varphi\left(v_{n}\right)\right) \\
& \quad \leq 0 .
\end{aligned}
$$

The choice of $h_{l}$ and $l>k$ implies

$$
\begin{aligned}
& \int_{\left\{l<\left|\varphi\left(v_{n}\right)\right|<l+1\right\}} \sigma h_{l}^{\prime}\left(\varphi\left(v_{n}\right)\right)\left(T_{k} \varphi\left(v_{n}\right)-\left(T_{k} \varphi(v)\right)_{\nu}\right) \nabla \varphi\left(v_{n}\right) \cdot \nabla \varphi\left(v_{n}\right) \\
& \quad \geq-2 k \int_{\left\{l<\left|\varphi\left(v_{n}\right)\right|<l+1\right\}} \sigma\left|\nabla \varphi\left(v_{n}\right)\right|^{2} .
\end{aligned}
$$


Choose the test function $\sigma \phi\left(\varphi\left(v_{n}\right)\right)$, where $\phi_{l}(r)=\operatorname{sign}_{0}(r)(|r|-l)^{+} \wedge 1$, we get

$$
\lim _{l \rightarrow \infty} \sup _{n} \int_{\left\{l<\left|\varphi\left(v_{n}\right)\right|<l+1\right\}}\left|\nabla \varphi\left(v_{n}\right)\right|^{2} \leq 0
$$

Further

$$
\begin{aligned}
& \int_{\left\{\varphi\left(v_{n}\right) \geq k\right\}} \sigma h_{l}\left(\varphi\left(v_{n}\right)\right) \nabla \varphi\left(v_{n}\right) \cdot \nabla T_{k} \varphi\left(v_{n}\right)=0 \\
& \limsup _{\nu \rightarrow \infty} \limsup _{n \rightarrow \infty} \int_{\left\{\varphi\left(v_{n}\right) \geq k\right\}} \sigma h_{l}\left(\varphi\left(v_{n}\right)\right) \nabla \varphi\left(v_{n}\right) \cdot \nabla\left(T_{k} \varphi(v)\right)_{\nu} \\
& \leq \int_{\{|w| \geq k\}} \sigma h_{l}(w) \nabla T_{k+1} w \cdot \nabla T_{k} w=0 .
\end{aligned}
$$

Hence, as $l \rightarrow \infty$, it results from (5.23) that

$$
\limsup _{\nu \rightarrow \infty} \limsup _{n \rightarrow \infty} \int_{Q} \sigma \nabla T_{k} \varphi\left(v_{n}\right) \cdot \nabla\left(T_{k} \varphi\left(v_{n}\right)-\left(T_{k} \varphi(v)\right)_{\nu}\right) \leq 0 .
$$

As a further consequence,

$\limsup _{\nu \rightarrow \infty} \limsup _{n \rightarrow \infty} \int_{Q} \sigma\left(\nabla T_{k} \varphi\left(v_{n}\right)-\nabla\left(T_{k} \varphi(v)\right)_{\nu}\right) \cdot \nabla\left(T_{k} \varphi\left(v_{n}\right)-\left(T_{k} \varphi(v)\right)_{\nu}\right)=0$.

By a diagonal principle, there exists a sequence $n(\nu)$ such that the function $\sigma\left|\nabla T_{k} \varphi\left(v_{n}\right)-\nabla\left(T_{k} \varphi(v)\right)_{\nu}\right|^{2}$ converges to zero strongly in $L^{1}(Q)$ as $\nu \rightarrow \infty$. We deduce that

$$
\nabla T_{k} \varphi\left(v_{n(\nu)}\right) \cdot \nabla\left(T \varphi\left(v_{n(\nu)}\right)-\left(T_{k} \varphi(v)\right)_{\nu}\right) \rightarrow 0 \text { weakly in } L^{1}(Q)
$$

and then, by using the fact that $\nabla T_{k} \varphi\left(v_{n(\nu)}\right) \rightarrow \nabla T_{k} \varphi(v)$ weakly in $L^{1}(Q)$ as $\nu \rightarrow \infty$, that

$$
\sigma\left|\nabla T_{k} \varphi\left(v_{n(\nu)}\right)\right|^{2} \rightarrow \sigma\left|\nabla T_{k} \varphi(v)\right|^{2} \quad \text { weakly in } L^{1}(Q) \text { as } \nu \rightarrow \infty .
$$

Estimate (5.18) then follows.

Now, let us pass to the limit in (4.13) with $n \rightarrow \infty$. Take $h\left(\varphi\left(v_{n}\right)\right) \xi$, where $h \in C_{c}^{1}(\mathbb{R}), \xi \in C_{c}^{\infty}([0, T) \times \Omega)$ as a test function in inequality (4.13), and pass to the limit with $n$ in each term. By means of the dominated convergence theorem, we conclude that

$$
\lim _{n \rightarrow \infty} \int_{Q} f_{n} h\left(\varphi\left(v_{n}\right)\right) \xi=\int_{Q} f h(w) \xi
$$

and

$$
\lim _{n \rightarrow \infty} \int_{Q} \psi_{n}\left(\varphi\left(v_{n}\right)\right) h\left(\varphi\left(v_{n}\right)\right) \xi=0
$$

Lemma 2.1 implies

$$
\int_{Q} j\left(v_{n}\right)_{t} h\left(\varphi\left(v_{n}\right)\right) \xi=-\int_{Q} \xi_{t} \int_{j\left(v_{n}^{0}\right)}^{j\left(v_{n}\right)} h\left(\varphi \circ j_{0}^{-1}\right)(r) d r
$$


and by means of the dominated convergence theorem again, we have

$$
\lim _{n \rightarrow \infty} \int_{Q} \xi_{t} \int_{j\left(v_{n}^{0}\right)}^{j\left(v_{n}\right)} h\left(\varphi \circ j_{0}^{-1}\right)(r) d r=-\int_{Q} \xi_{t} \int_{u_{0}}^{u} h\left(\varphi \circ j_{0}^{-1}\right)(r) d r .
$$

From (5.18), and the fact that $j\left(v_{n}\right) \rightarrow u$ a.e. on $Q$ and $\varphi\left(v_{n}\right) \rightarrow w$ a.e. on $Q$ we deduce that

$$
\begin{aligned}
& \lim _{n \rightarrow \infty} \int_{Q}\left(\nabla \varphi\left(v_{n}\right)+F\left(j\left(v_{n}\right), \varphi\left(v_{n}\right)\right)\right) \cdot \nabla\left(h\left(\varphi\left(v_{n}\right)\right) \xi\right) \\
& \quad=\int_{Q}(\nabla w+F(u, w)) \cdot \nabla(h(w) \xi) .
\end{aligned}
$$

Remains to prove that $u$ satisfies $(2.4)$. For this aim, take $T_{l+1}\left(\varphi\left(v_{n}\right)\right)-$ $T_{l}\left(\varphi\left(v_{n}\right)\right)$ as a test function in (5.15). Thanks again to Lemma 2.1 and the monotonicity of the function $\psi_{n}$, we have

$$
\begin{aligned}
& \int_{Q \cap\left\{l \leq\left|\varphi\left(v_{n}\right)\right| \leq l+1\right\}}\left\{\left|\nabla \varphi\left(v_{n}\right)\right|^{2}+F\left(j\left(v_{n}\right), \varphi\left(v_{n}\right)\right) \cdot \nabla \varphi\left(v_{n}\right)\right\} \\
& \quad \leq \int_{Q \cap\left\{\left|\varphi\left(v_{n}\right)\right| \geq l\right\}}\left|f_{n}\right|+\int_{\left\{\left|v_{n}^{0}\right| \geq l\right\}}\left|v_{n}^{0}\right| .
\end{aligned}
$$

Passing to the limit as $n \rightarrow \infty$ and arguing as for (3.5) to prove that $\int_{\left\{l<\left|\varphi\left(v_{n}\right)\right|<l+1\right\}} F\left(j\left(v_{n}\right), \varphi\left(v_{n}\right)\right) \cdot \nabla \varphi\left(v_{n}\right)=0$, we get

$$
\limsup _{n \rightarrow \infty} \int_{Q \cap\left\{l \leq\left|\varphi\left(v_{n}\right)\right| \leq l+1\right\}}\left|\nabla \varphi\left(v_{n}\right)\right|^{2} \leq \int_{Q \cap\{|w| \geq l\}}|f|+\int_{\Omega \cap\left\{\left|v_{0}\right| \geq l\right\}}\left|v_{0}\right| .
$$

So, since $\left|\nabla \varphi\left(v_{n}\right)\right|^{2} \chi_{\left\{l<\left|\varphi\left(v_{n}\right)\right|<l+1\right\}}=\left|\nabla\left(T_{l+1} \varphi\left(v_{n}\right)-T_{l} \varphi\left(v_{n}\right)\right)\right|^{2}$ and $T_{l+1}$ $\varphi\left(v_{n}\right)-T_{l} \varphi\left(v_{n}\right) \rightarrow T_{l+1} w-T_{l} w$ weakly in $L^{2}\left(0, T ; H_{0}^{1}(\Omega)\right)$, then

$$
\int_{Q \cap\{l \leq|w| \leq l+1\}}|\nabla w|^{2} \leq \int_{Q \cap\{|w| \geq l\}}|f|+\int_{\Omega \cap\left\{\left|v_{0}\right| \geq l\right\}}\left|v_{0}\right|
$$

and, letting $l \rightarrow \infty$, we obtain

$$
\iint_{\{l \leq|w| \leq l+1\}}|\nabla w|^{2} \rightarrow 0 \text { as } l \rightarrow \infty .
$$

Finally, collecting together all limits (5.24)-(5.28) we conclude on existence of a renormalized solution of Problem $E\left(u_{0}, f\right)$ for all $f \in L^{1}(Q)$ and $u_{0} \in$ $L^{1}(\Omega)$.

\section{Proof of Theorem 2.1: Existence part.}

Let $G$ be the map from $[0, T) \times L^{1}(\Omega)$ into $L^{1}(\Omega)$ defined by

$$
G(t, u)=g(t, \cdot, u),
$$

and $A$ be the operator in $L^{1}(\Omega)$, defined by

$$
A z=-\Delta \varphi(z)-\nabla F(z, w), w \in \beta(z) \text { in } \mathcal{D}^{\prime}(\Omega)
$$

and

$$
D(A)=\left\{z \in L^{\infty}(\Omega) ; w \in H_{0}^{1}(\Omega), A z \in L^{1}(\Omega)\right\} .
$$


Thanks to [15], we know that $A$ is $T$-accretive in $L^{1}(\Omega)$ and $\bar{A}$ is $m$-accretive in $L^{1}(\Omega)$, and, moreover, $\overline{D(A)}=L^{1}(\Omega)$.

Thanks to $i$ ) and $i i)$ of Assumption $\left(H_{3}\right), G$ is integrable in $t \in(0, T)$ for any $u \in L^{1}(\Omega)$ and continuous in $u \in L^{1}(\Omega)$ for a.e. $t \in(0, T)$. Moreover, using $\left.i i\right)$ of Assumption $\left(H_{3}\right)$ we see that $C I-G(t,$.$) is accretive in L^{1}(\Omega)$. Then (see for instance [9], Lemma 1) there exists a unique mild solution of

$$
\frac{d u}{d t}+A u=G(\cdot, u) \text { on }(0, T), \quad u(0)=u_{0}
$$

which is also a mild solution of

$$
\frac{d u}{d t}+A u \ni f, u(0)=u_{0}
$$

with $f=g(\cdot, u)$. By Proposition $4.2, u$ is a renormalized solution of $E\left(u_{0}, f\right)$ and thus $u$ is a renormalized solution of $E\left(u_{0}, g\right)$.

\section{The elliptic problem}

At the end of this paper, let us give some consequences of the previous results for the stationary problem

$$
\begin{cases}u-\Delta w-\nabla F(u, w)=f, w \in \beta(u) & \text { in } \Omega \\ w=0 & \text { on } \partial \Omega\end{cases}
$$

by assuming that Assumptions $\left(H_{1}\right)-\left(H_{2}\right)$ are fulfilled.

Proposition 6.1. Let $f \in L^{1}(\Omega)$. Then, there exists a unique renormalized solution $u$ of $S(f)$ in the sense that

(i) $u \in L^{1}(\Omega)$

(ii) $T_{k} w \in H_{0}^{1}(\Omega)$ for any $k>0$

(iii) for all $\xi \in \mathcal{D}(\Omega)$ and $h \in \mathcal{C}_{c}^{1}(\mathbb{R})$

$$
\int_{\Omega}(u-f) h(w) \xi+\int_{\Omega}(\nabla w+F(u, w)) \cdot \nabla(h(w) \xi)=0,
$$

and moreover

$$
\int_{\Omega \cap\{n \leq|w| \leq n+1\}}|\nabla w|^{2} \rightarrow 0 \text { as } n \rightarrow \infty .
$$

Moreover, for any $f_{i} \in L^{1}(\Omega)$ and $u_{i}$ a renormalized solution of $S\left(f_{i}\right), i=1,2$, we have

$$
\left\|\left(u_{1}-u_{2}\right)^{+}\right\|_{1} \leq\left\|\left(f_{1}-f_{2}\right)^{+}\right\|_{1} .
$$

Proof. The uniqueness follows from the fact that if $u$ is a renormalized solution of $S(f)$ then $\tilde{u}(t) \equiv u$ is a renormalized solution of $E\left(\tilde{u}_{0}, \tilde{g}\right)$ with $\tilde{u}_{0}=u$ and $\tilde{g}(\cdot, u)=f(\cdot)-u$. 
To prove existence, we consider a sequence $f_{n}$ in $L^{\infty}(\Omega)$ such that $f_{n}$ converges to $f$ in $L^{1}(\Omega)$ as $n \rightarrow \infty$. It follows from [15] that there exists a unique $u_{n}$ solution of

$$
\left\{\begin{array}{l}
u_{n} \in L^{\infty}(\Omega), w_{n} \in \beta\left(u_{n}\right) \in H_{0}^{1}(\Omega) \text { and } \\
u_{n}=\nabla \cdot\left(\nabla w_{n}+F\left(u_{n}, w_{n}\right)\right)+f_{n} \text { in } \mathcal{D}^{\prime}(\Omega)
\end{array}\right.
$$

moreover, we have

$$
\left\|u_{n}-u_{m}\right\|_{L^{1}(\Omega)} \leq\left\|f_{n}-f_{m}\right\|_{L^{1}(\Omega)} \quad \text { for any } n, m \in \mathbb{N} .
$$

This implies that $\left(u_{n}\right)_{n \in \mathbb{N}}$ is a Cauchy sequence in $L^{1}(\Omega)$ and there exists $u \in L^{1}(\Omega)$ such that $u_{n} \rightarrow u$ in $L^{1}(\Omega)$ as $n \rightarrow \infty$. To prove that $u$ is a renormalized solution of $S(f)$, note that $u_{n}$ is also a renormalized solution of the evolution problem $E\left(u_{n}, f_{n}-u_{n}\right)$; therefore, passing to the limit as $n \rightarrow \infty$, the result follows.

Corollary 6.1. The closure of the operator $A$ in $L^{1}(\Omega)$ satisfies

$$
\begin{aligned}
\bar{A} & =\left\{(z, h) \in L^{1}(\Omega) \times L^{1}(\Omega) ; z \text { is a renormalized solution of } S(u+h)\right\} \\
& =: \mathcal{A} .
\end{aligned}
$$

Proof. Since a weak solution is also a renormalized solution, we have $A \subseteq \mathcal{A}$. On the other hand, using Theorem 5.1 , we deduce that $\mathcal{A}$ is $m$-accretive in $L^{1}(\Omega)$, so that $\mathcal{A}$ is closed in $L^{1}(\Omega)$, and

$$
\bar{A} \subseteq \mathcal{A} .
$$

Thanks to [15], we know that $A$ is accretive and $R(I+A) \supseteq L^{\infty}(\Omega)$, then $\bar{A}$ is $m$-accretive in $L^{1}(\Omega)$, and (6.29) implies that $\bar{A}=\mathcal{A}$.

\section{Appendix}

Lemma 7.1. Let $h \in W^{1, \infty}(\mathbb{R}), h \geq 0, u_{0} \in L^{1}(\Omega), u \in L^{1}(Q)$ such that $T_{k} w \in L^{2}\left(0, T ; H_{0}^{1}(\Omega)\right)$ for any $k>0$ and $G \in L^{2}\left(0, T ; H^{-1}(\Omega)\right)+L^{1}(Q)$. Suppose that

$$
\int_{Q} \xi_{t} \int_{u_{0}}^{u} h\left(\varphi_{0}(s)\right) d s=\int_{0}^{T}\langle G, h(w) \xi\rangle
$$

for any nonnegative $\xi \in \mathcal{D}([0, T) \times \Omega)$. Then,

$$
\iint_{Q} \xi_{t}\left\{\int_{u_{0}}^{u} H_{\varepsilon}\left(T_{k} \varphi_{0}(s)-\varphi(z)\right) h\left(\varphi_{0}(s)\right) \mathrm{d} s\right\} \leq \int_{0}^{T}\left\langle G, H_{\varepsilon}\left(T_{k} w-\varphi(z)\right) h(w) \xi\right\rangle .
$$

for all $\xi \in L^{2}\left(0, T ; H^{1}(\Omega)\right) \cap W^{1,1}\left(0, T ; L^{\infty}(\Omega)\right) \cap L^{\infty}(Q)$ such that $\xi \geq 0$, $\xi(T,)=$.0 a.e. in $\Omega$, and for any $z \in L^{1}(Q)$ such that $\varphi(z) \xi \in L^{2}\left(0, T ; H_{0}^{1}(\Omega)\right)$. 
Proof. We extend $u$ onto $\mathbb{R} \times \Omega$ by 0 if $t>T$ and by $u_{0}$ if $t<0$ and we consider

$$
\Phi=H_{\varepsilon}\left(T_{k} w-\varphi(z)\right) \xi .
$$

It is clear that $\Phi \in L^{2}\left(0, T ; H_{0}^{1}(\Omega)\right)$ and, for any $\delta>0, \Phi^{\delta}(t)=\frac{1}{\delta} \int_{t}^{t+\delta} \Phi(s) \mathrm{d} s$ is an admissible test function in the problem (7.30) and

$$
\iint_{Q} \Phi_{t}^{\delta} \int_{u_{0}}^{u} h\left(\varphi_{0}(s)\right) d s=\int_{0}^{T}\left\langle G, \Phi^{\delta} h(w)\right\rangle .
$$

We see that

$$
\begin{aligned}
\iint_{Q} \Phi_{t}^{\delta} \int_{u_{0}}^{u} h\left(\varphi_{0}(s)\right) d s & =\iint_{Q} \frac{\Phi(t+\delta)-\Phi(t)}{\delta} \int_{u_{0}}^{u} h\left(\varphi_{0}(s)\right) d s \\
& =\iint_{Q} \Phi(t) \frac{1}{\delta} \int_{u(t)}^{u(t-\delta)} h\left(\varphi_{0}(s)\right) d s
\end{aligned}
$$

and, since for any $r, \hat{r}, w \in \mathbb{R}$,

$$
H_{\varepsilon}\left(T_{k} \varphi_{0}(r)-\varphi(w)\right) \int_{r}^{\hat{r}} h\left(\varphi_{0}(s)\right) d s \leq \psi_{w}^{\varepsilon}(\hat{r})-\psi_{w}^{\varepsilon}(r),
$$

where $\psi_{w}^{\varepsilon}(r)=\int_{w}^{r} H_{\varepsilon}\left(T_{k} \varphi_{0}(s)-\varphi(w)\right) h\left(\varphi_{0}(s)\right) \mathrm{d} s$, it follows that

$$
\begin{aligned}
& \iint_{Q} \Phi_{t}^{\delta} \int_{u_{0}}^{u} h\left(\varphi_{0}(s)\right) d s \leq \iint_{Q} \frac{\psi_{z(t, x)}^{\varepsilon}(u(t-\delta, x))-\psi_{z(t, x)}^{\varepsilon}(u(t, x))}{\delta} \xi(t, x) \mathrm{d} t \mathrm{~d} x \\
& \leq \iint_{Q}\left(\psi_{z(t, x)}^{\varepsilon}(u(t, x))-\psi_{z(t, x)}^{\varepsilon}\left(u_{0}(x)\right)\right) \frac{\xi(t+\delta, x)-\xi(t, x)}{\delta} \mathrm{d} t \mathrm{~d} x .
\end{aligned}
$$

Consequently, we have

$$
\begin{aligned}
\liminf _{\delta \rightarrow 0} \iint_{Q} \Phi_{t}^{\delta} \int_{u_{0}}^{u} h\left(\varphi_{0}(s)\right) d s & \leq \iint_{Q}\left(\psi_{z}^{\varepsilon}(u)-\psi_{z}^{\varepsilon}\left(u_{0}\right)\right) \xi_{t} \\
& \leq \iint_{Q} \xi_{t}\left\{\int_{u_{0}}^{u} H_{\varepsilon}\left(T_{k} \varphi_{0}(s)-\varphi(z)\right) \mathrm{d} s\right\} .
\end{aligned}
$$

Since $h\left(T_{k} w\right) \Phi^{\delta} \rightarrow H_{\varepsilon}\left(T_{k} w-\varphi(z)\right) h(w) \xi$ in $L^{2}\left(0, T ; H_{0}^{1}(\Omega)\right)$, then from (7.32) and the preceding estimate, (7.31) follows.

\section{References}

[1] Alt, H.W., Luckhaus, H.W.: Quasilinear elliptic-parabolic differential equations. Math. Z. 183, 311-341 (1983)

[2] Ammar, K., Andreu, F., Toledo, J.: On a quasi-linear elliptic problem in $L^{1}$ with non homogeneous boundary conditions. Rend. Mat. Serie VII 26, 291-314 (2006)

[3] Ammar, K., Wittbold, P.: Existence of renormalized solutions of degenerate elliptic-parabolic problems. Proc. Roy. Soc. Edinburgh A 133(3), 477-496 (2003)

[4] Andreianov, B., Igbida, N.: Revising uniqueness for a nonlinear diffusion convection equation. J. Differ. Equ. 227, 69-79 (2006) 
[5] Andreianov, B., Sbihi K., Wittbold, P.: On uniqueness and existence of entropy solutions for a nonlinear parabolic problem with absorption (Submitted)

[6] Andreu, F., Igbida, N., Mazon, J.M., Toledo, J.: Renormalized solutions for degenerate elliptic-parabolic problems with nonlinear dynamical boundary conditions and $L^{1}$-data (Submitted)

[7] Bénilan, Ph.: Équation d'évolution dans un espace de banach quelconque et applications. Thesis, Orsay (1972)

[8] Bénilan, Ph., Crandall, M.G., Pazy, A.: Evolutions Equations Governed by Accretive Operators. Preprint book

[9] Bénilan, Ph., Igbida, N.: Singular limit for perturbed nonlinear semigroup. Comm. Appl. Nonlinear Anal. 3, 23-42 (1996)

[10] Bénilan, Ph., Wittbold, P.: On mild and weak solutions of elliptic-parabolic problems. Adv. Differ. Equ. 1, 1053-1073 (1996)

[11] Blanchard, D., Murat, F.: Renormalized solutions of nonlinear parabolic problems with $L^{1}$ data: existence and uniqueness. Proc. Roy. Soc. Edinburgh A 127, 1137-1152 (1997)

[12] Blanchard, D., Porretta, B.: Stefan problems with nonlinear diffusion and convection. J. Differ. Equ. 210, 383-428 (2005)

[13] Blanchard, D., Redwane, H.: Solutions renormalisées d'équations paraboliques à deux non linéaritées. C. R. Acad. Sci. Paris 319, 831-847 (1994)

[14] Boccardo, L., Giachetti, D., Diaz, J.I., Murat, F.: Existence of solutions for a weaker form of a nonlinear elliptic equation. In: Pitman Res. Notes Math. Ser. 208, editor. Recent Advances in Nonlinear Elliptic and Parabolic Problems, pp. 229-246. Longman Sc. Tech., Harlow (1989)

[15] Carrillo, J.: Entropy solutions for nonlinear degenerate problems. Arch. Rat. Mech. Anal. 147, 269-361 (1999)

[16] Carrillo, J., Wittbold, P.: Uniqueness of renormalized solutions of degenerate elliptic-parabolic problems. J. Differ. Equ. 1, 93-121 (1999)

[17] Damlamian, A.: Some result on the multiphase Stefan problem. Comm. Part. Differ. Equ. 2, 1017-1044 (1977)

[18] Di Perna, R.J., Lions, P.L.: On the Cauchy problem for Boltzmann equations: global existence and weak stability. Ann. Math. 130, 321-366 (1989)

[19] Friedman, A.: Variational Principles and Free-boundary Problems. Wiley, New York (1982)

[20] Gagneux, G., Madaune-Tort, M.: Analyse mathématique de modèles non linéaires de l'ingénierie pétrolière. Mathématiques \& Applications, Springer, Berlin (1996)

[21] Igbida, N., Urbano, J.M.: Uniqueness for nonlinear degenerate problems. NoDEA Nonlinear Differ. Equ. Appl. 10(3), 287-307 (2003) 
[22] Kobayasi, K.: The equivalence of weak solutions and entropy solutions of nonlinear degenerate second-order equations. J. Differ. Equ. 189, 383-395 (2003)

[23] Kačur, J.: On a solution of degenerate elliptic-parabolic systems in orlicz-sobolev spaces i-ii. Math. Z. 203, 153-171 (1990)

[24] Landes, R.: On the existence of weak solutions for quasilinear parabolic initial boundary-value problems. Proc. Roy. Soc. Edinburgh A 89, 217-237 (1981)

[25] Murat, F.: Soluciones renormalizadas de EDP elípticas no lineales. Publ. Laboratoire d'Analyse Numérique Univ. Paris 6, R 93023 (1993)

[26] Otto, F.: $L^{1}$-contraction and uniqueness for quasilinear elliptic-parabolic equations. J. Differ. Equ. 131, 20-38 (1996)

[27] Rakotoson, J.M.: Generalized solutions in a new type of sets for problems with measures as data. Differ. Int. Equ. 6, 27-36 (1993)

[28] Sbihi, K.: Etude de quelques E.D.P. non linéaires dans $L^{1}$ avec des conditions générales sur le bord. PhD. Thesis Strasbourg (2006)

[29] Sbihi, K., Wittbold, P.: Existence de solutions renormalisées pour un problème de Stefan non linéaire. C. R. Acad. Sci. Paris Ser. I 345, 629-632 (2007)

[30] Tarzia, D.: A bibliography on moving-free boundary problems for the heatdiffusion equation. The Stefan and related problems. M.A.T. Seria A: Mathematical Conferences, Seminar and Papers, vol. 2 (2000)

[31] Urbano, J.M.: A free boundary problem with convection for the $p$-Laplacian. Rend. Math. Appl. 17, 1-19 (1997)

[32] Urbano, J.M.: Continuous solutions for a degenerate free boundary problem. Ann. Math. Pura Appl. 178, 195-224 (2000)

N. Igbida

LAMFA, CNRS-UMR 6140,

Université de Picardie Jules Verne,

33 rue Saint Leu,

80038 Amiens,

France

e-mail: noureddine.igbida@u-picardie.fr

K. Sbihi

Laboratoire de Mathématiques,

Université de Franche-comté,

16 route de Gray,

25030 Besancon,

France

e-mail: karima.sbihi@univ-fcomte.fr 
P. Wittbold

Technische Universität Berlin,

Strasse des 17. Juni 136,

10623 Berlin,

Germany.

e-mail: wittbold@math.tu-berlin.de

Received: 09 November 2008.

Accepted: 16 September 2009. 